\title{
Mechanism of tacrolimus-induced chronic renal fibrosis following transplantation is regulated by ox-LDL and its receptor, $\mathrm{LOX}-1$
}

\author{
SHI DENG ${ }^{1 *}$, TAO JIN ${ }^{1 *}$, LI ZHANG ${ }^{2}$, HONG BU $^{3}$ and PENG ZHANG ${ }^{1}$ \\ ${ }^{1}$ Department of Urology, Institute of Urology; ${ }^{2}$ Key Laboratory of Transplant Engineering and Immunology, \\ Ministry of Health; ${ }^{3}$ Department of Pathology, West China Hospital, \\ Sichuan University, Chengdu, Sichuan 610041, P.R. China
}

Received May 31, 2015; Accepted June 27, 2016

DOI: $10.3892 / \mathrm{mmr} .2016 .5735$

\begin{abstract}
Chronic renal allograft dysfunction (CRAD) is the most common cause of graft failure following renal transplantation. However, the underlying mechanisms remain to be fully elucidated. Immunosuppressants and hyperlipidemia are associated with renal fibrosis following long-term use. The present study aimed to determine the effects of tacrolimus (FK506) and lipid metabolism disorder on CRAD. In vitro and in vivo models were used for this investigation. Cells of the mouse proximal renal tubular epithelial cell strain, NRK-52E, were cultured either with oxidized low-density lipoprotein (ox-LDL), FK506, ox-LDL combined with FK506, or vehicle, respectively. Changes in cell morphology and changes in the levels of lectin-like ox-LDL receptor-1 (LOX-1), reactive oxygen species (ROS), hydrogen peroxide and fibrosis-associated genes were evaluated at 24,48 and $72 \mathrm{~h}$. In separate experiment, total of 60 Sprague-Dawley rats were divided randomly into four groups, which included a high-fat group, FK506 group, high-fat combined with FK506 group, and control group. After 2, 4 and 8 weeks, the serum lipid levels, the levels of ox-LDL, ROS, and the expression levels of transforming growth factor (TGF)- $\beta 1$ and connective tissue growth factor were determined. The in vitro and in vivo models revealed that lipid metabolism disorder and FK506 caused oxidative stress and a fibrogenic response. In addition, decreased levels of LOX-1 markedly reduced the levels of TGF- $\beta 1$ in the in vitro
\end{abstract}

Correspondence to: Professor Peng Zhang, Department of Urology, Institute of Urology, West China Hospital, Sichuan University, 37 Guoxuexiang Street, Chengdu, Sichuan 610041, P.R. China

E-mail: zhangpenghuaxi@163.com

*Contributed equally

Key words: kidney transplantation, tacrolimus, chronic renal allograft dysfunction, hyperlipidemia, oxidative stress, renal fibrosis model. Taken together, FK506 and dyslipidemia were found to be associated with CRAD following transplantation.

\section{Introduction}

As one of the most effective treatments against end-stage renal disease, renal allografts markedly improve patient survival rates and quality of life (1-3). With developments in transplantation immunobiology and the application of novel immunosuppressants, numerous problems, including acute rejection and short-term graft loss, have been addressed, resulting in decreased mortality rates (2). However, improvements in long-term graft survival are not commensurate. With further prolongation of the transplantation period, renal allografts gradually lose their function (3). This irreversible, progressive loss is generally termed chronic renal allograft dysfunction (CRAD) (1), which is caused by immunological and non-immunological factors (2). As a non-immunological factor, dyslipidemia leads to cardiovascular disease (CVD) and severely impairs the long-term survival of grafts and patients (4-6). Of note, 40-80\% patients have been reported to exhibit hyperlipidemia following renal transplantation $(3,7)$.

Low-density lipoprotein (LDL) is critical in lipid metabolism, as is its bioactive oxidized form, oxidized low-density lipoprotein (ox-LDL). Lectin-like ox-LDL receptor-1 (LOX-1), the major receptor of ox-LDL, is a key molecule in vascular endothelial function disorder due to atherosclerosis, which can accelerate the development of CRAD following renal transplantation (8-10). In the current clinical setting, tacrolimus (FK-506) is widely used as a selective immunosuppressor to improve the short-term survival rates of grafts and patients. However, with further prolongation of the transplantation period, its toxic effect aggravates CRAD and can even increase long-term mortality rates (11). Although several reports have focussed on this side effect, the underlying mechanism remains to be elucidated. Current knowledge is limited to the suggestion that lipid peroxidation and excess reactive oxygen species (ROS) production caused by oxidative stress are crucial to this toxic function (12).

Hyperlipidemia is closely associated with CRAD (13). The early pathological changes of atherosclerosis are similar to 
those of CRAD, and CRAD is sometimes considered another form of atherosclerosis, induced by immune injury and aggravated by immunological and non-immunological factors. Metabolic syndrome is common in transplant patients, and it is largely attributed to the use of immunosuppressant agents, such as calcinurin inhibitors and prednisone. FK-506 is associated with a lower incidence of hypertension and hypercholesterolaemia than cyclosporine. However, 10-30\% of FK-506 users still develop hypercholesterolemia $(14,15)$, and the higher the tacrolimus trough level, the higher the rate of hypercholesterolemia (16). FK-506 is the most widely used calcineurin inhibitor in transplantation. It is associated with a complicated side effect profile, including post-transplant diabetes mellitus, dyslipidemia and toxicity; which eventually result in allograft fibrosis. However, the exact mechanism underlying this effect is unclear. Therefore, the present study aimed to investigate whether renal fibrosis caused by FK506 occurs via its effects on high lipid levels; this effect was mimicked by administering a high fat diet or inducing high ox-LDL levels.

The present study used in vitro and in vivo models, which were exposed to FK506 and high lipid levels to observe the expression levels of ox-LDL, LOX-1 and certain chronic fibrosis-associated genes, including transforming growth factor- $\beta$ (TGF- $\beta$ ) and connective tissue growth factor (CTGF), to determine the effects of FK506 and high lipid levels on CRAD.

\section{Materials and methods}

Cells and animals. The mouse proximal renal tubular epithelial cell strain, NRK-52E, was purchased from the American Type Culture Collection (Manassas, VA, USA). Healthy male Sprague-Dawley rats 6-8 weeks weighing 180-220 g were provided by the Experimental Center of Sichuan University (Sichuan, China). Animals were fed and maintained at $20-22^{\circ} \mathrm{C}$ in a $12 \mathrm{~h}$ light/dark cycle, with access to standard chow and water ad libitum. Animal experimentation was performed according to the Ethical Committee of Sichuan University.

Cell experiments. Thawed NRK-52E cells were sustained in 60-mm dishes with high-glucose Dulbecco's modified Eagle's medium (DMEM; Gibco, Thermo Fisher Scientific, Inc. Waltham, MA, USA) supplemented with $10 \%$ fetal bovine serum, $4 \mathrm{mmol} / 1$ glutamine, $100 \mu \mathrm{g} / \mathrm{ml}$ streptomycin and $100 \mathrm{IU} / \mathrm{ml}$ penicillin at $37^{\circ} \mathrm{C}$ in $5 \% \mathrm{CO}_{2}$. The culture medium was refreshed 2-3 days later and the cells were passaged on day 4-5 at a ratio of 1:3. The cells were then cultured in different treatment groups, as follows: $80 \mu \mathrm{g} / \mathrm{ml}$ ox-LDL; $50 \mu \mathrm{g} / \mathrm{ml}$ FK506; $80 \mu \mathrm{g} / \mathrm{ml}$ ox-LDL+50 $\mu \mathrm{g} / \mathrm{ml} \mathrm{FK506;} \mathrm{and}$ vehicle, respectively. The expression of LOX-1 was determined using immunofluorescence. The levels of ROS and hydrogen peroxide were analyzed using ROS and hydrogen peroxide test kits (Nanjing Jiancheng Bioengineering Institute, Nanjing, China), according to the manufacturer's protocol. Total RNA was isolated using the SV Total RNA isolation system (Promega Corporation, Madison, WI, USA), according to the manufacturer's protocol. mRNA levels were determined using reverse transcription-quantitative polymerase chain reaction (RT-qPCR) and proteins levels were determined using western blotting. In addition, three fluorescent-labeled small interfering (si)RNAs (Guangdong Ruibo Biotechnology Co., Ltd., Guangdong, China) were designed specifically against LOX-1, and were transfected into the NRK-52E cells.

Animal experiments. A total of 60 Sprague-Dawley rats were divided randomly into four treatment groups, which included a high-fat group, FK506 group, high-fat+FK506 group and control group. The FK506 rats were fed with common chow supplemented with $0.15 \mathrm{mg} / \mathrm{kg} /$ day FK506. The rats in the high-fat+FK506 group were fed a high-fat diet (which consists of $40 \%$ lard, $5 \%$ milk powder, $5 \%$ peanut flour, $5 \%$ sesame powder and $40 \%$ general fodder) in addition to supplementation with $0.15 \mathrm{mg} / \mathrm{kg} /$ day FK506. Access to water and food were unrestricted in all experiments. The rats were anaesthetized with an intraperitoneal injection of $10 \%$ chloral hydrate $(35 \mathrm{mg} / \mathrm{kg})$. The eyeballs of five randomly-selected rats in each group at weeks 2, 4 and 8 were extracted to collect blood samples $(1.5 \mathrm{ml})$. Then the serum levels of total cholesterol, triglycerides, LDL cholesterol and ox-LDL were analyzed. Following blood lipid analyses, the randomly selected rats were sacrificed by decapitation prior to kidney isolation and the remaining unselected rats were sacrificed by high level of $\mathrm{CO}_{2}$. The isolated kidney was divided in half, one of which was maintained in liquid nitrogen for further analyses, including RT-qPCR analysis and western blotting, whereas the other was fixed in $4 \%$ formalin for $24-48 \mathrm{~h}$ for pathological analysis and immunohistochemistry.

Immunofluorescence. The expression of LOX-1 was determined using immunofluorescence. Briefly, cells were cultured on cover slips in 6-well plates. When the cells were grown to $>80 \%$ confluence, the cells were fixed in $4 \%$ paraformaldehyde for $10 \mathrm{~min}$. The cells were then washed with Tris-buffered saline with $0.1 \%$ Tween (TBST) twice and blocked in $1.5 \%$ bovine serum albumin (Beyotime Institute of Biotechnology, Shanghai, China)/TBST for $1 \mathrm{~h}$ at room temperature. Then cells were incubated with primary antibodies against LOX-1 (1:100; cat no. DY1564; Santa Cruz Biotechnology Inc., Dallas, TX, USA) overnight at $4^{\circ} \mathrm{C}$. After washing in TBST 3 times, cells were incubated with fluorescein isothiocyanate-conjugated goat anti-rabbit immunoglobulin G (1:100; cat no. 2729S; Santa Cruz Biotechnology Inc.) for $1 \mathrm{~h}$ and stained with DAPI for $5 \mathrm{~min}$. The images were viewed and recorded by Olympus BX40 and SPOT Flex 4.6 Prelim/4.6.0.0. (Diagnostic Instruments, Thermo Fisher Scientific, Inc.).

ROS and $\mathrm{H}_{2} \mathrm{O}_{2}$ measurement. The levels of ROS and $\mathrm{H}_{2} \mathrm{O}_{2}$ in NRK-52E cells were analyzed using ROS and $\mathrm{H}_{2} \mathrm{O}_{2}$ test kits (Nanjing Jiancheng Bioengineering Institute, Nanjing, China), according to the manufacturer's protocol. The Superoxide Anion Assay kit (BYJC0022, Nanjing Jiancheng Bioengineering Institute, Nanjing, China) was used to simulate the reaction between xanthine and xanthine oxidase in organisms. It produces the superoxide anion $\operatorname{radical~}_{2} \bullet$, which results in a purple coloring when Griess reagent is added. This was measured at optical density (OD)550 using a spectrophotometer (SmartSpec ${ }^{\text {TM }}$ 3000; Bio-Rad Laboratories, Inc., Hercules, CA, USA). The influence of the checked samples to $\mathrm{O}_{2}$ can be 
Table I. siRNA sequences used in the present study.

\begin{tabular}{|c|c|c|c|}
\hline Name & Serial number & Target sequence & siRNA sequence \\
\hline $\begin{array}{l}\text { SiLOX-1_001 } \\
\text { (NM_133306) }\end{array}$ & siB0822282236 & CTGGAAGCTAAACGAGAAA & $\begin{array}{l}\text { Sense: 5'-CUGGAAGCUAAACGAGAAA dTdT-3' } \\
\text { Antisense: } 3^{\prime} \text {-dTd TGACCUUCGAUUUGCUCUUU-5' }\end{array}$ \\
\hline $\begin{array}{l}\text { SiLOX-1_002 } \\
(\text { NM_13306) }\end{array}$ & siB0822282305 & GCAGAATCAGAACCTCCAA & $\begin{array}{l}\text { Sense: 5'-GCAGAAUCAGAACCUCCAA dTdT-3' } \\
\text { Antisense: 3'-dTdT CGUCUUAGUCUUGGAGGUU-5' }\end{array}$ \\
\hline $\begin{array}{l}\text { SiLOX-1_003 } \\
(\text { NM_13306) }\end{array}$ & siB0822282327 & GGAGAATTGCCTATCTTTA & $\begin{array}{l}\text { Sense: 5'-GGAGAAUUGCCUAUCUUUA dTdT-3' } \\
\text { Antisense: 3'-dTdT CCUCUUAACGGAUAGAAAU-5' }\end{array}$ \\
\hline
\end{tabular}

siRNA, small interfering RNA.

calculated using VitC as the reference. The complex, which was produced by $\mathrm{H}_{2} \mathrm{O}_{2}$ and the molybdic acid, was examined by measuring OD405 utilizing the Hydrogen Peroxide Assay kit (K265-200, Nanjing Jiancheng Bioengineering Institute, Nanjing, China).

Reverse transcription-quantitative polymerase chain reaction $(R T-q P C R)$. Total RNA was isolated using the SV Total RNA isolation system (Promega Corporation, Madison, WI, USA), according to the manufacturer's protocol. To compare LOX-1, TGF- $\beta 1$ and CTGF mRNA expression in different experimental groups, RNA samples were isolated from cells or kidney tissue using TRIzol reagent (Invitrogen; Thermo Fisher Scientific, Inc.). First strand cDNA synthesis was conducted using the cDNA synthesis kit (Toyobo Co., Ltd., Osaka, Japan). qPCR was performed with SYBR Green PCR Master mix on the CFX96 Touch PCR system (Applied Biosystems, Thermo Fisher Scientific, Inc.). The reaction consisted of $5 \mu \mathrm{l}$ of $2 \mathrm{X}$ SYBR Green mix, $1 \mu \mathrm{l}$ cDNA, $1 \mu 1$ of each primer at a concentration of $10 \mu \mathrm{mol} / \mathrm{ml}$ and $3 \mu \mathrm{l}$ of $\mathrm{ddH}_{2} \mathrm{O}$ mixed gently in each well of the Applied Biosystems MicroAmp Optical 96-Well Reaction Plate. Reactions were performed under the following conditions: Initial denaturation at $95^{\circ} \mathrm{C}$ for $30 \mathrm{sec}$ (one cycle), followed by denaturation at $95^{\circ} \mathrm{C}$ for $15 \mathrm{sec}$ and annealing/extension at $60^{\circ} \mathrm{C}$ for $30 \mathrm{sec}(40 \mathrm{cycles})$, with a final melt curve cycle of $5 \mathrm{sec}$ at $65^{\circ} \mathrm{C}$. The endogenous Glyceraldehyde 3-phosphate dehydrogenase (GAPDH) gene was used as the internal control for promoter screening. The relative RNA expression was calculated with the comparative $\mathrm{Cq}$ method, which was normalized to the internal references (17). Data were analyzed using CFX Manager Software v3.1 Upgrader (Applied Biosystems, Thermo Fisher Scientific, Inc.).-The primers used were as follows: Forward: 5'-GAACGCTTCAGAGGAGTCCA-3' and reverse: 5'-AGGCAATTCTCCCGACTTTT-3' for LOX-1; forward: 5'-TGAGTGGCTGTCTTTTGACG-3' and reverse: 5'-TTCTCTGTGGAGCTGAAGCA-3' for TGF- $\beta 1$; forward: 5'-AAGACACATTTGGCCCTGAC-3' and reverse primer 5'-CCACAGAACTTAGCCCGGTA-3' for CTGF; and forward: 5'-ACCACAGTCCATGCCATCAC-3' and reverse: 5'-TCCACCACCCTGTTGCTGTA-3' for GAPDH.

Western blot analysis. Levels of LOX-1 and TGF- $\beta 1$ proteins in cells and tissues were determined using western blotting. Cells or kidney tissue were lysed with radioimmunoprecipitation assay buffer in (50 mM Tris base, $1.0 \mathrm{mM}$ EDTA, $150 \mathrm{mM} \mathrm{NaCl}, 0.1 \%$ SDS, $1 \%$ Triton X-100, $1 \%$ sodium deoxycholate and $1 \mathrm{mM}$
PMSF). The protein sample was separated by $10 \%$ SDS-PAGE electrophoresis, and then transferred from the gel onto the polyvinylidene difluoride membrane. After blocking with 5\% nonfat milk in TBST for $1 \mathrm{~h}$ at $37^{\circ} \mathrm{C}$, the membrane was incubated with the following primary antibodies: Goat anti-LOX-1 polyclonal antibody (1:1;000; cat no. DY1564; Santa Cruz Biotechnology Inc.) and mouse anti-TGF- $\beta 1$ monoclonal antibody $(1: 1,000$; cat. no. MAB240; R\&D Systems, Minneapolis, MN, USA) and rabbit monoclonal anti-GAPDH antibody (1:5,000; cat. no. ab181602, Abcam, Cambridge, CA, USA) at $4^{\circ} \mathrm{C}$ overnight. The next day, membranes were washed in phosphate-buffered saline (PBS) with $0.1 \%$ Tween-20 for 15 min, three times. The membranes were then incubated with horseradish peroxidase (HRP)-conjugated donkey anti-goat (1:1,000; cat. no. A0181) and goat anti-mouse (1:1,000; cat. no. A0216) secondary antibodies (Beyotime Institute of Biotechnology) at $37^{\circ} \mathrm{C}$ for $1 \mathrm{~h}$. Detection was performed with enhanced chemiluminescence (Amersham Biosciences UK Ltd., Little Chalfont, UK) and bands were quantified using ImageJ v2.1.4.7 (National Institutes of Health, Bethesda, MD, USA). GAPDH was used as a control and was detected using anti-GAPDH antibodies (1:5,000; cat no. ab181602; Abcam).

Small interfering (si)RNAs against LOX-1 in NRK-52e cells. Three fluorescent-labeled siRNAs (Guangdong Ruibo Biotechnology Co., Ltd., Guangdong, China) were designed specifically against LOX-1, and were transfected into the NRK-52E cells (sequences are listed in Table I) Briefly, 2.5-3x $10^{4}$ cells per well were plated in 24-well plates. The following day, 50 pmol FAM-siRNA was combined with $1.5 \mu 1$ Lipofectamine 2000 transfection reagent (Invitrogen, Thermo Fisher Scientific, Inc.) plus $100 \mu 1$ of Opt-MEM. The control RNA duplex was used to ensure that parallel experiments had equal amounts of RNA. After $6 \mathrm{~h}$ of culture at $37^{\circ} \mathrm{C}$, fluorescence expression was observed under a fluorescence microscope in 4 fields per well and repeat in 3 different wells. Transfection efficiency was calculated using flow cytometry (Bio-Rad Labotatories, Inc.) to determine the transfection efficiency according to the manufacturer's instructions using the following equation: \% Transfection efficiency $=$ (number of cells stained with fluorescent positive control dye/total number of cells per field) x 100. All experiments were conducted with 3 parallel duplicates.

Pathological analysis. The kidney tissues were fixed in $4 \%$ formaldehyde and embedded in paraffin. They were cut into $4-\mu \mathrm{m}$ slices and stained with hematoxylin and eosin and 
Masson's trichrome stain. For Masson's trichrome stain, the collagen stained green, muscle fibers stained red and blood cells stained orange. Images were viewed under a light microscope and analyzed using the Leica DMR+Q550 system (Leica Microsystems GmbH, Wetzlar, Germany).

Immunological analysis. LOX-1, TGF- $\beta 1$ and CTGF proteins were detected and localized by immunohistochemical staining. Kidney tissue sections $(4 \mu \mathrm{m})$ were deparaffinized in xylene and dehydrated with graduated ethanol solutions and antigen retrieval was performed. Briefly, $3 \% \mathrm{H}_{2} \mathrm{O}_{2}$ was used to block the endogenous peroxidase activity for $20 \mathrm{~min}$ at room temperature away from light. The tissues were then incubated with the following primary antibodies for $18 \mathrm{~h}$ at $4^{\circ} \mathrm{C}$ : Anti-LOX-1 (1:200, Sigma-Aldrich, St. Louis, MO, USA), anti-TGF- $\beta 1$ (1:400; Wuhan Boster Biological Technology, Ltd., Wuhan, China), and anti-CTGF (sc-1493; 1:200 Santa Cruz Biotechnology Inc.). The slides were then washed with PBS, and incubated with DAB. The images were gathered through Nikon spot cool CCD (Nikon, Tokyo, Japan) and the staining results were analyzed by the multimedia color pathological image analysis system (Mais2000-P3; LEIKE SI China Electric appliance Co., Ltd., Hubei, China).

Statistical analysis. For analysis of the immunohistochemical expression of each protein, ROS level, lipid level and kidney damage between all treatment groups, the nonparametric Kruskal-Wallis (analysis of variance) test was used. Analysis between specific pairs of treatment groups was compared using the Mann-Whitney unpaired t-test. For comparisons before and after treatment, a two-tailed paired t-test was used. Statistical software SPSS version 17.0 (SPSS Software, Inc., Chicago, IL, USA) was used for statistical analysis. $\mathrm{P}<0.05$, was considered to indicate a statistically significant difference.

\section{Results}

Changes in cell morphology and levels of LOX-1 in NRK-52E cells following treatment with FK506 and ox-LDL. At $48 \mathrm{~h}$ post-ox-LDL treatment, a number of cells showed vacuolized cytoplasms and enlarged extracellular spaces, whereas cells in the FK506 group showed marked changes, with ground bodies, enlarged extracellular spaces and an increased proportion of floating dead cells. In addition to the majority of the dual-treated cells exhibiting the morphology of the other two groups, these cells also appeared aggregated and fragmented (Fig. 1). The immunofluorescence results showed that LOX-1 was expressed $24 \mathrm{~h}$ following administration in all groups, and reached the highest level at $48 \mathrm{~h}$ (Fig. 2). The expression level of LOX-1 in the ox-LDL group was 1.9-fold higher, compared with that in control group, whereas the level in the FK506 group was 1.4-fold higher, and that in the dual-treated group was 2.3-fold higher, compared with the control group. The latter was highest among all groups $(\mathrm{P}<0.01)$.

Levels of ROS and hydrogen peroxide increase in NRK-52E cells following treatment with FK506 and ox-LDL. The cells in all treatment groups were assessed using the ROS and hydrogen peroxide test kits at 24, 48 and $72 \mathrm{~h}$. The levels of ROS and hydrogen peroxide increased at $24 \mathrm{~h}$, and reached the highest levels at $48 \mathrm{~h}$, prior to decreasing thereafter. The levels of ROS and hydrogen peroxide in the three treated groups were higher, compared with those in the control group, and the levels in the dual-treated group were the highest overall $(\mathrm{P}<0.01)$.

Levels of LOX-1,TGF- $\beta 1$ and CTGF in NRK-52E cells following treatment with FK506 and ox- $L D L$. At $48 \mathrm{~h}$ post-treatment, the mRNA levels of $L O X-1, T G F-\beta 1$ and $C T G F$, determined using RT-qPCR, were significantly increased $(\mathrm{P}<0.01)$, and were highest in the dual-treated group $(\mathrm{P}<0.01$; Fig. $3 \mathrm{~A})$. The results of the western blotting to examine the protein levels of LOX-1 and TGF- $\beta 1$ were similar, with the levels of LOX-1 and TGF- $\beta 1$ increasing significantly following treatment $(\mathrm{P}<0.01)$. The highest levels of these proteins were observed in the dual-treated cells $(\mathrm{P}<0.01$; Fig. 3B).

Decreased LOX-1 decreases the expression of TGF- $\beta 1$. In the present study, NRK-52E cells were transfected with three FAM-labeled siRNAs against LOX-1, and the levels of TGF- $\beta 1$ in these cells were determined using flow cytometry $48 \mathrm{~h}$ following transfection. The decrease in LOX-1 markedly reduced the levels of TGF- $\beta 1$. The levels of TGF- $\beta 1$ were significantly decreased following transfection with any of the siRNAs $(\mathrm{P}<0.01)$, with siLOX-1_001 transfection resulting in the most marked reduction.

Changes in serum lipid and ox-LDL levels in rats following treatment with FK506 and a high-fat diet. After 2 weeks on a high-fat diet combined with FK506, the serum levels of total cholesterol, triglycerides and LDL cholesterol in the rats were significantly increased $(\mathrm{P}<0.01)$. This increase was enhanced at weeks 4 and 8, whereas the level of high-density lipoprotein cholesterol were significantly decreased $(\mathrm{P}<0.01)$. In all the treated rats, those in the high-fat diet+FK506 had the highest serum lipid levels $(\mathrm{P}<0.01)$. The increase in serum levels of ox-LDL, determined using ELISA, were significant in all the treated rats at 2 weeks $(\mathrm{P}<0.01)$, and this was more marked at 4 and 8 weeks $(\mathrm{P}<0.01)$. These levels were highest in the rats in the high-fat diet+FK506 group. Significant differences were observed between the three groups $(\mathrm{P}<0.05)$.

Levels of ROS and rat kidney fibrogenesis increase following treatment with FK506 and a high-fat diet. The levels of ROS and hydrogen peroxide in the rat kidneys increased in a time-dependent manner, and the highest levels were observed in rats in the high-fat diet+FK506 group $(\mathrm{P}<0.01)$. The rat kidneys were isolated for H\&E and Masson's trichrome staining at 2, 4 and 8 weeks. The results of the H\&E staining showed that, in the kidneys treated for 2 weeks, the renal tubules showed marginal swelling, with hemorrhaging of the tubulointerstitial substance and glomeruli. At 4 weeks, the renal tubule swelling became more marked, whereas the degree of hemorrhage declined and hyperplasia was observed. At 8 weeks, necrosis and atrophy were present in certain tubules and glomeruli, and bleeding of the tubulointerstitial substance remained (Fig. 4). In the Masson's trichrome staining, green tubular and tubulointerstitial fibrogenesis was observed, which increased over time (Fig. 5). Compared with the controls, the degrees of fibrogenesis at weeks 2, 4 and 8 increased by 2.1 -, 3.2- and 5.3-fold, respectively $(\mathrm{P}<0.01)$. 

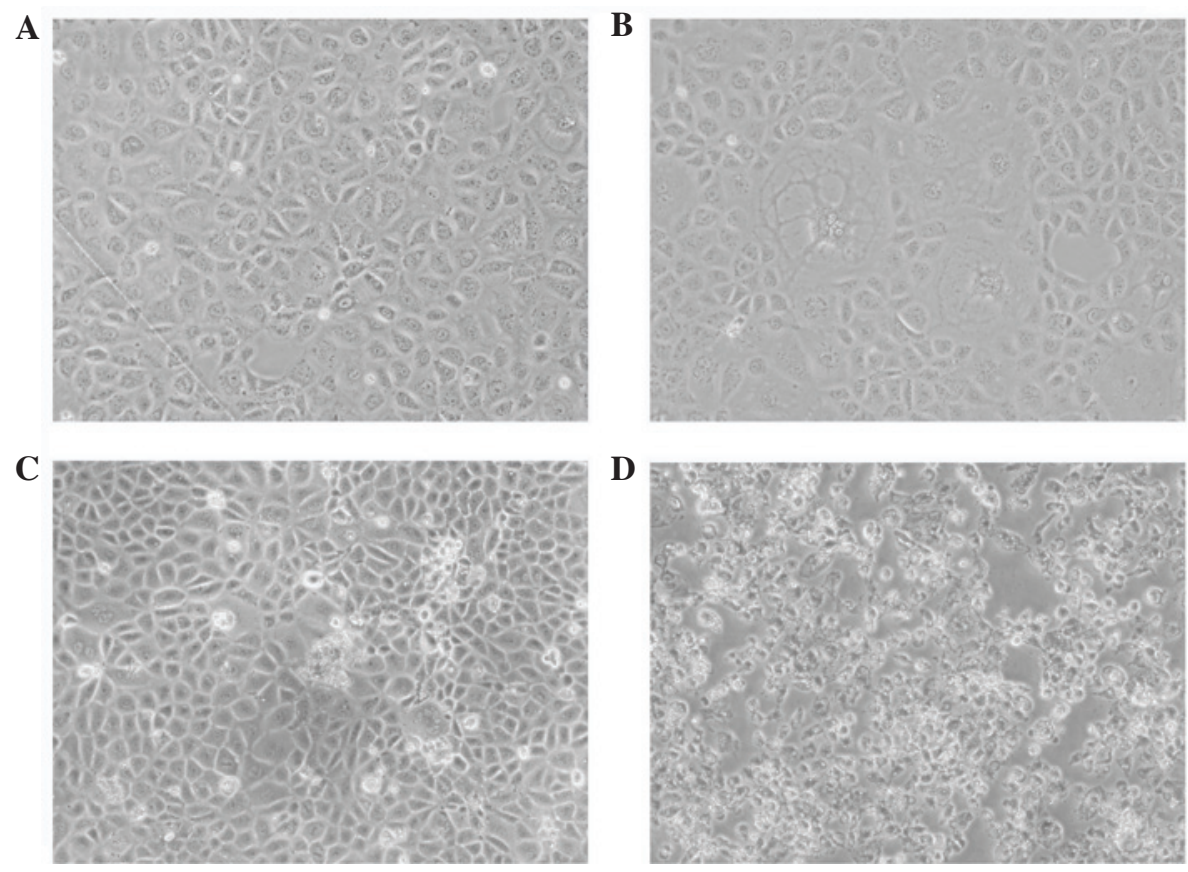

Figure 1. Changes in NRK-52E cell morphology following treatment with FK506 and ox-LDL. Variation in the morphology of NRK-52E cells of the (A) control group, (B) ox-LDL group $(80 \mu \mathrm{g} / \mathrm{ml}$ for $48 \mathrm{~h}$ ), (C) FK506 group $(50 \mu \mathrm{g} / \mathrm{ml}$ for $48 \mathrm{~h}$ and (D) ox-LDL+FK506 group $(80 \mu \mathrm{g} / \mathrm{ml}$ ox-LDL $+50 \mu \mathrm{g} / \mathrm{ml}$ FK506 for 48 h). Original magnification, x200. ox-LDL, oxidized low-density lipoprotein; FK506, tacrolimus.
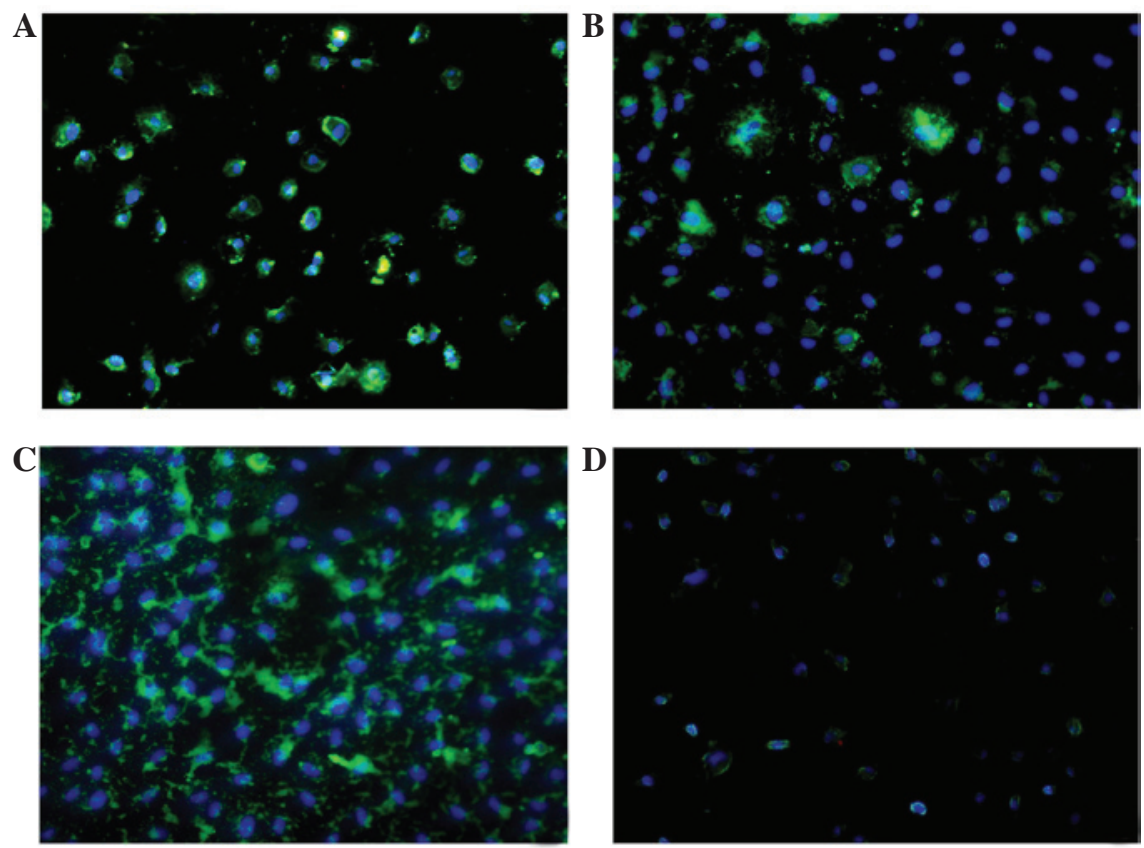

Figure 2. Changes in levels of LOX-1 in NRK-52E cells following treatment with FK506 and ox-LDL. Immunofluorescence showing the expression of lectin-like ox-LDL receptor-1 in the (A) ox-LDL group $(80 \mu \mathrm{g} / \mathrm{ml}$ for $48 \mathrm{~h}$ ). (B) FK506 group (50 $\mu \mathrm{g} / \mathrm{ml}$ for $48 \mathrm{~h}$ ). (C) ox-LDL+FK506 group ( $80 \mu \mathrm{g} / \mathrm{ml}$ ox-LDL+50 $\mu \mathrm{g} / \mathrm{ml}$ FK506 for 48 h) and (D) control group. Original magnification, x200. ox-LDL, oxidized low-density lipoprotein; FK506, tacrolimus.

The increase in the degree of fibrogenesis at 8 weeks was significantly higher, compared with the increases at 2 and 4 weeks $(\mathrm{P}<0.05)$.

Levels of LOX-1, TGF- $\beta 1$ and CTGF are increased in rats following treatment with FK506 and a high-fat diet. The results of the immunohistochemical analyses showed that LOX-1 was expressed at low levels in the glomeruli and tubules following 2 weeks of treatment, which increased over time, and was highest in the rats in the high-fat diet+FK506 rats (Fig. 6). Compared with the control, the levels of LOX-1 in the rats of the high-fat diet+FK506 group were 1.5-, 2.0- and 3.1-fold higher at 2, 4 and 8 weeks, respectively $(\mathrm{P}<0.01)$. The levels of TGF- $\beta 1$ and CTGF were also highest in this group of rats (Figs. 7 and 8). By contrast, the highest level of TGF- $\beta 1$ was observed at 4 weeks, which 

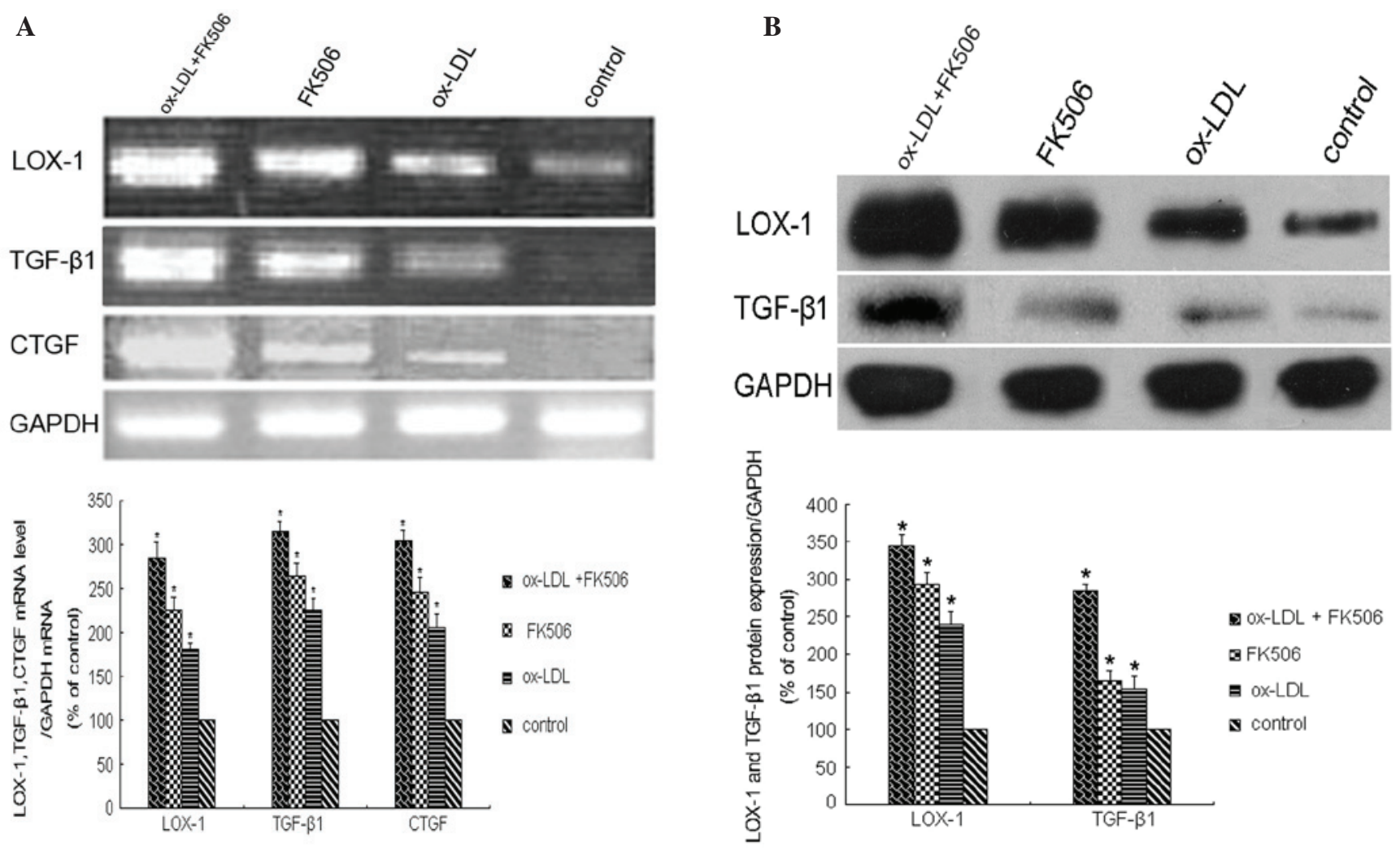

Figure 3. (A) mRNA levels of LOX-1, TGF- $\beta 1$ and CTGF in the cells of the ox-LDL+FK506 group ( $80 \mu \mathrm{g} / \mathrm{m}$ ox-LDL+50 $\mu \mathrm{g} / \mathrm{ml} \mathrm{FK506} \mathrm{for} 48 \mathrm{~h}$ ), ox-LDL group $(80 \mu \mathrm{g} / \mathrm{ml}$ for $48 \mathrm{~h})$ and FK506 group $(50 \mu \mathrm{g} / \mathrm{ml}$ for $48 \mathrm{~h})$. (B) Protein expression levels of LOX-1 and TGF- $\beta 1$ in the cells of the ox-LDL+FK506 group (80 $\mu \mathrm{g} / \mathrm{m}$ ox-LDL+50 $\mu \mathrm{g} / \mathrm{ml}$ FK506 for $48 \mathrm{~h}$ ), ox-LDL group $(80 \mu \mathrm{g} / \mathrm{ml}$ for $48 \mathrm{~h}$ ) and FK506 group (50 $\mu \mathrm{g} / \mathrm{ml}$ for $48 \mathrm{~h})$. "P $<0.01$ vs. control group ( 3 independent experiments). ox-LDL, oxidized low-density lipoprotein; FK506, tacrolimus; LOX-1, lectin-like ox-LDL receptor-1; TGF- $\beta 1$, transforming growth factor- $\beta 1$; CTGF connective tissue growth factor.

A
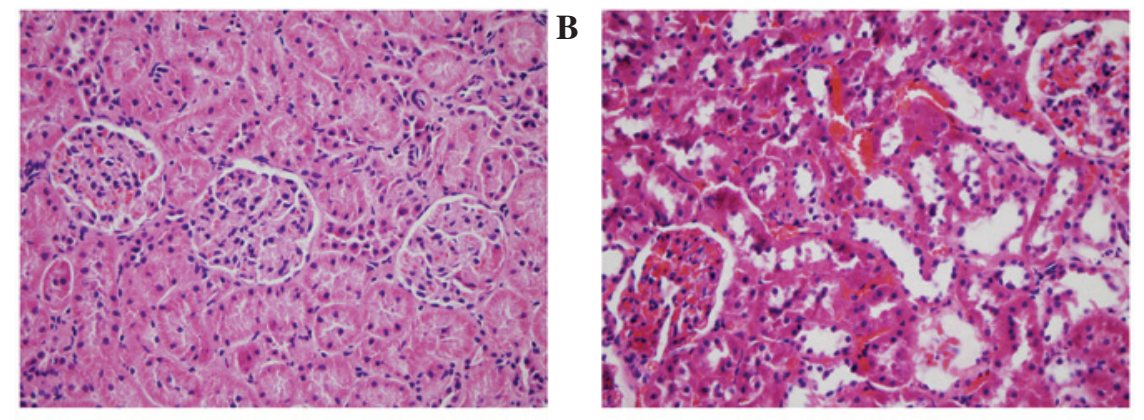

C

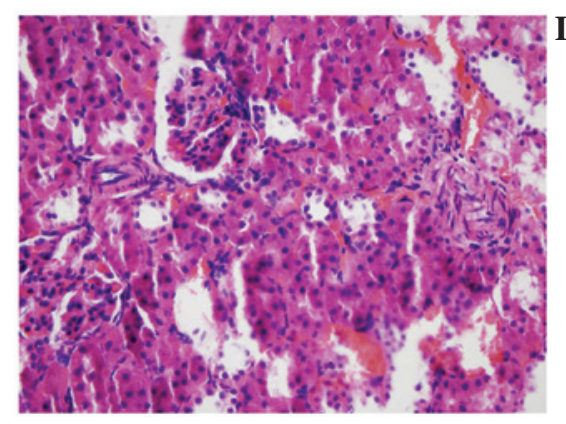

D

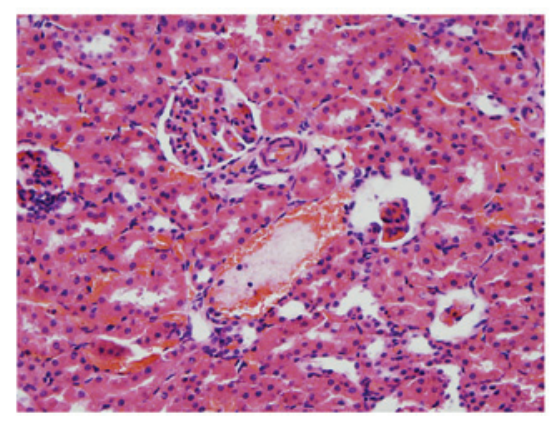

Figure 4. Hematoxylin and eosin staining of kidney tissues at different time points in the different groups of rats. (A) Control; (B) high fat diet+FK506 $(0.15 \mathrm{mg} / \mathrm{kg} /$ day) for 2 weeks; (C) high fat diet+FK506 (0.15 mg/kg/day) for 4 weeks; (D) high fat diet+FK506 (0.15 mg/kg/day) for 8 weeks. Original magnification, x400. FK506, tacrolimus.

subsequently decreased. Compared with the controls, these levels were increased by 1.5-, 1.9- and 1.3-fold at 2, 4 and
8 weeks, respectively $(\mathrm{P}<0.01)$. Similar to LOX-1, the levels of CTGF began to increase in the tubules at 2 weeks, and 

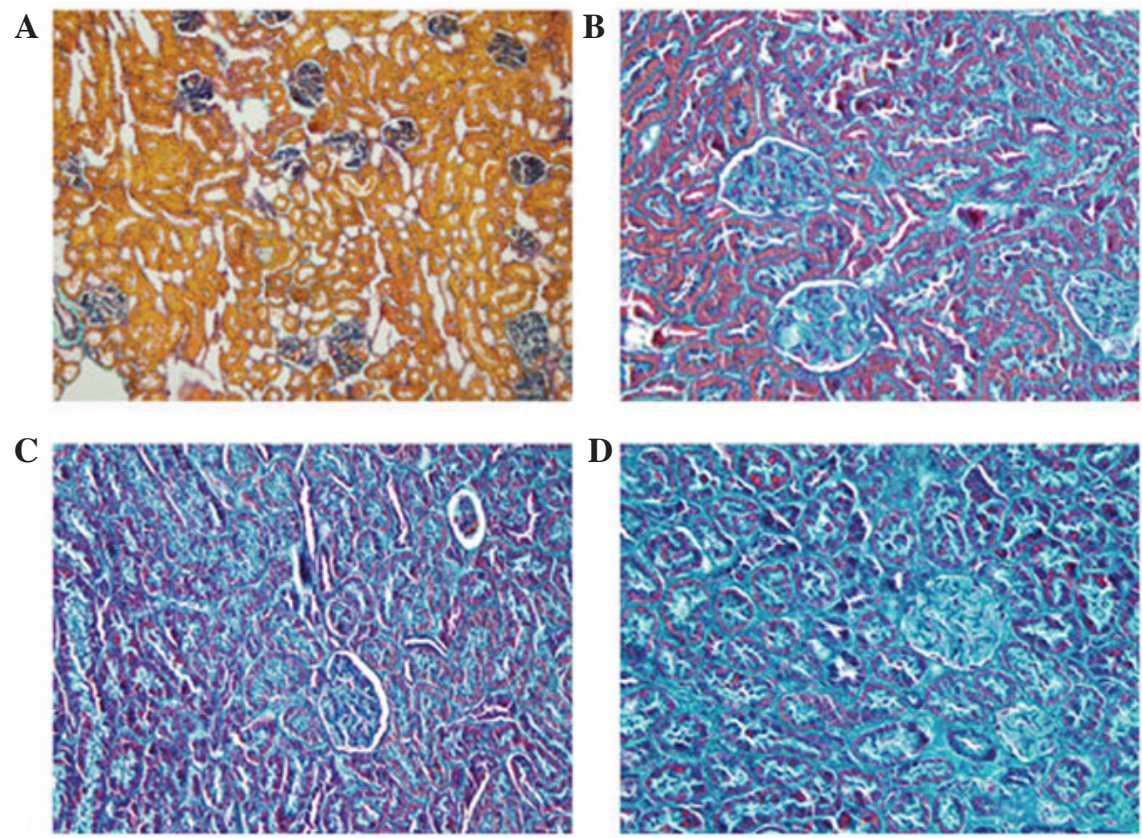

Figure 5. Masson's staining of fiber expression at different time points. (A) Control; (B) high fat diet+FK506 (0.15 mg/kg/day) for 2 weeks; (C) high fat diet+FK506 (0.15 mg/kg/day) for 4 weeks; (D) high fat diet+FK506 (0.15 mg/kg/day) for 8 weeks. Original magnification, x400. FK506, tacrolimus.
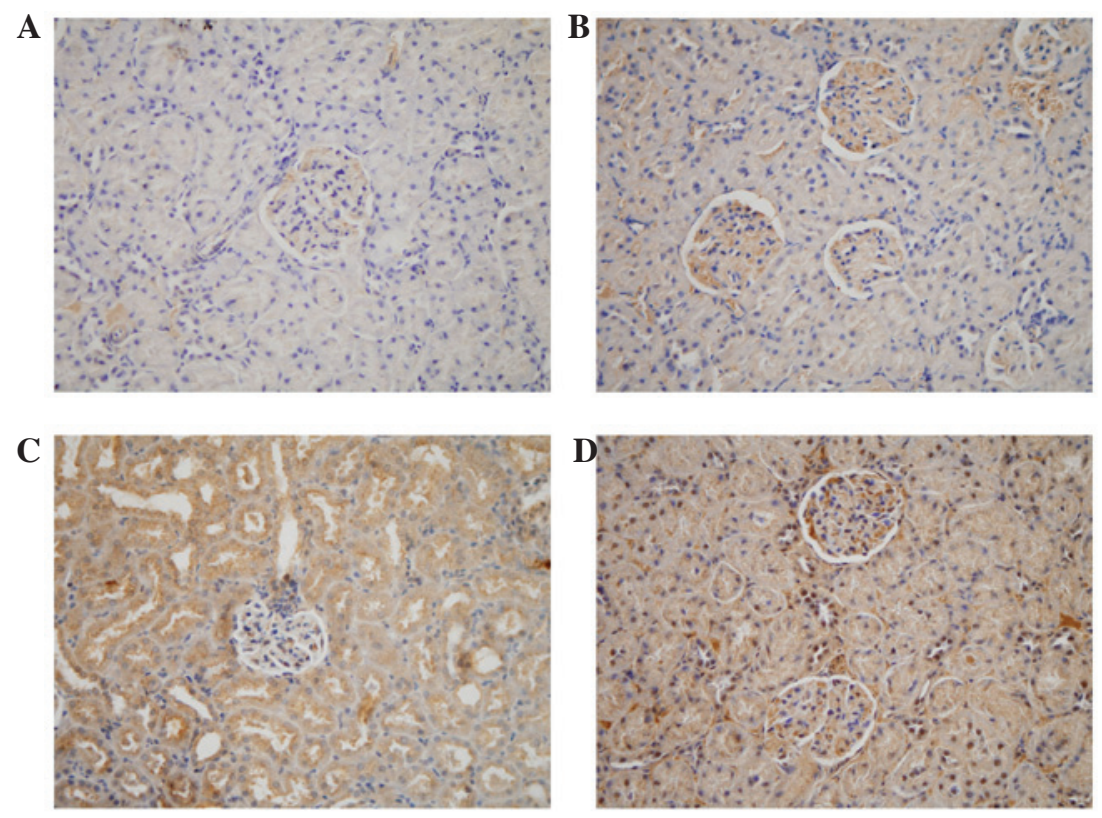

Figure 6. Expression of LOX-1 in the different groups, shown using immunohistochemistry. (A) Control; (B) high fat diet+FK506 (0.15 mg/kg/day) for 2 weeks; (C) high fat diet+FK506 (0.15 mg/kg/day) for 4 weeks; (D) high fat diet+FK506 (10 mg/kg/day) for 8 weeks. Original magnification, x400. FK506, tacrolimus;LOX-1, lectin-like oxidized low-density lipoprotein receptor-1.

continued to increase. At 8 weeks, CTGF was also expressed in areas of the glomeruli. The levels of CTGF increased 1.4-, 1.6- and 2-fold at 2, 4 and 8 weeks, respectively $(\mathrm{P}<0.01)$. At week 4 , the mRNA levels of $L O X-1, T G F-\beta 1$ and $C T G F$ were significantly increased $(\mathrm{P}<0.01)$, and were highest in the rats of the high-fat diet+FK506 group $(\mathrm{P}<0.01)$, and significantly different between the three treatment groups $(\mathrm{P}<0.05$; Fig. 9A). The protein levels of LOX- 1 and TGF- $\beta 1$ were also significantly increased $(\mathrm{P}<0.01)$, which were highest in the rats of the high-fat diet+FK506 group $(\mathrm{P}<0.01)$, and signifi- cantly different between the three treatment groups $(\mathrm{P}<0.05$; Fig. 9B).

\section{Discussion}

CRAD refers to renal hypofunction, which develops $\geq 3$ months following renal transplantation and leads to clinical findings of progressive serum creatinine increase, proteinuria and hypertension, and histological changes of vascular intimal hyperplasia, tubular atrophy, interstitial 

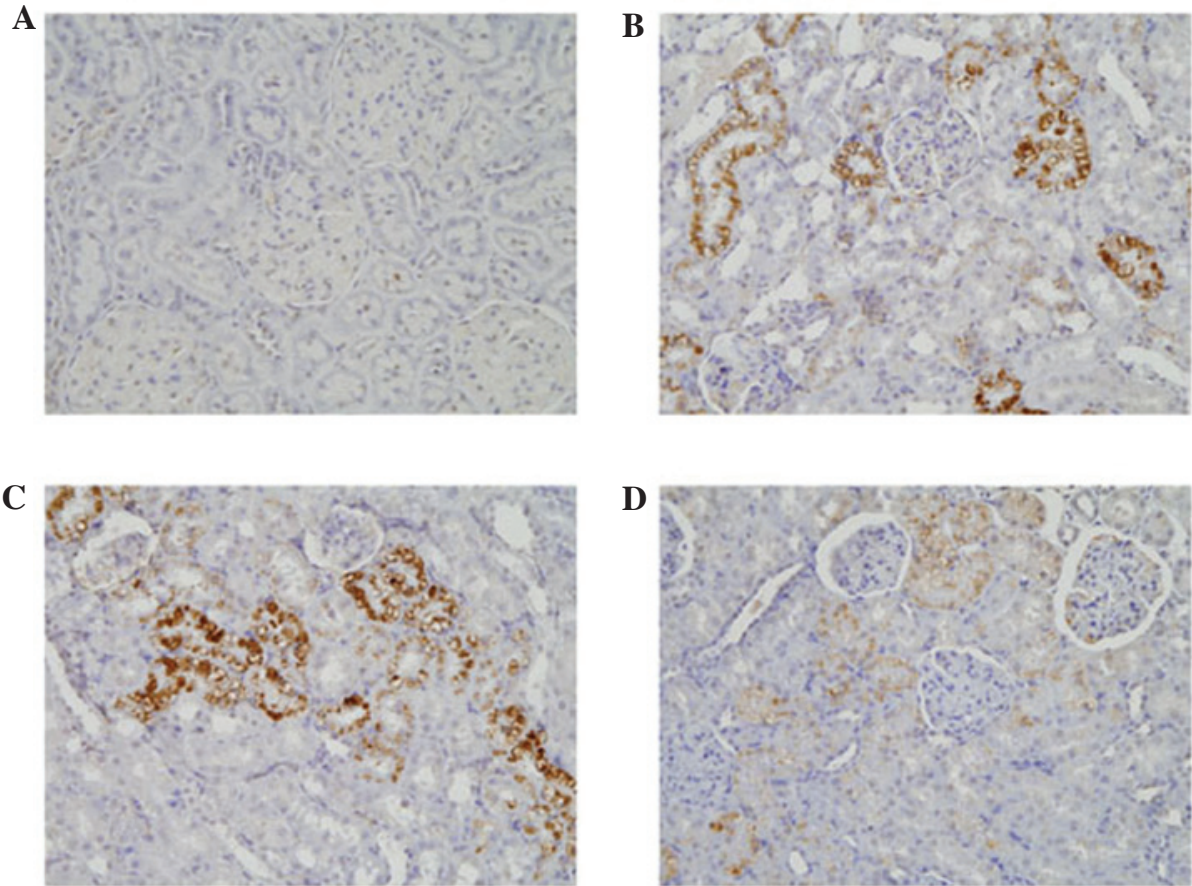

Figure 7. Expression of TGF- $\beta 1$ in different groups, analyzed using immunohistochemistry. (A) Control; (B) high fat diet+FK506 (0.15 mg/kg/day) for 2 weeks; (C) high fat diet+FK506 (0.15 mg/kg/day) for 4 weeks; (D) high fat diet+FK506 (0.15 mg/kg/day) for 8 weeks. Original magnification, x400. FK506, tacrolimus; TGF- $\beta 1$, transforming growth factor- $\beta 1$.

A

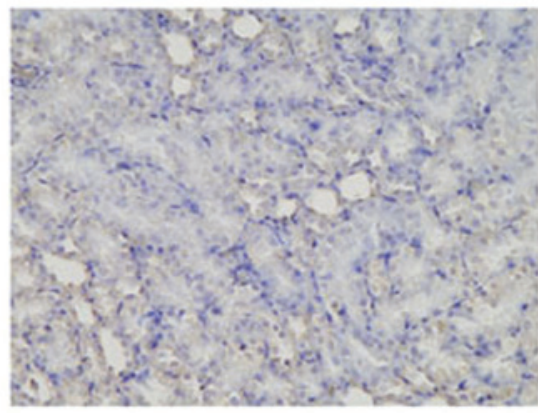

C

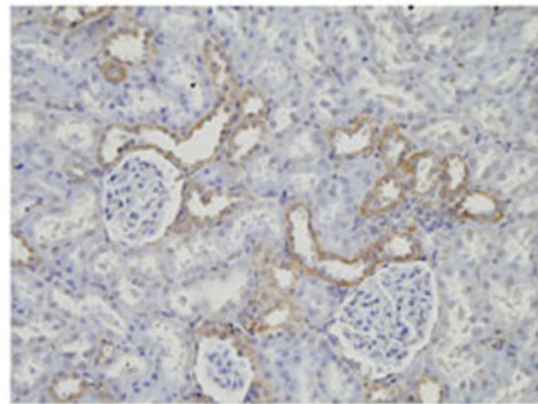

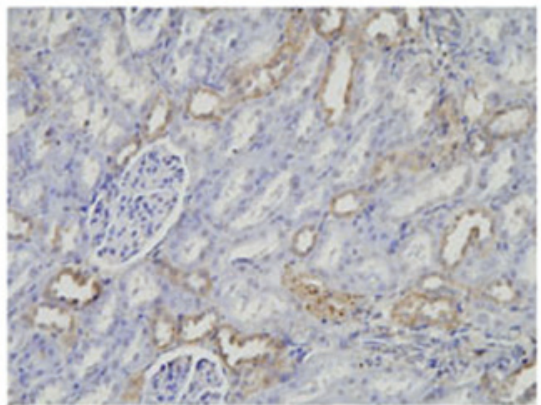

D

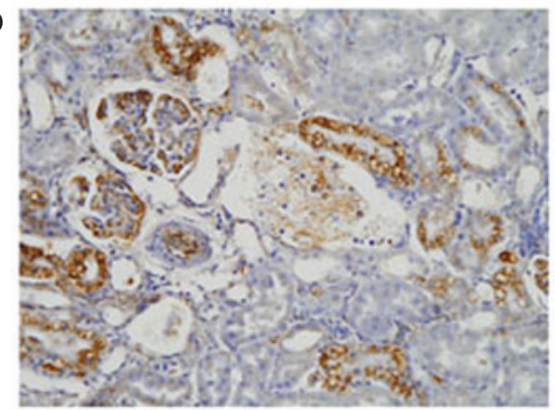

Figure 8. Expression of CTGF in different groups, analyzed using immunohistochemistry. (A) Control; (B) high fat diet+FK506 (0.15 mg/kg/day) for 2 weeks; (C) high fat diet + FK506 (0.15 mg/kg/day for 4 weeks; (D) high fat diet+FK506 (0.15 mg/kg/day) for 8 weeks. Original magnification, x400. FK506, tacrolimus; CTGF connective tissue growth factor.

fibrosis and chronic transplant glomerulopathy (8-13). Differential diagnoses that require exclusion include acute rejection, renal diseases and renal toxicity (18). As one of the typical characteristics of CRAD is interstitial fibrosis, and the early pathological changes of atherosclerosis are so similar, CRAD is sometimes considered to be another form of atherosclerosis, which is induced by immune injury and aggravated by immunological and non-immunological factors (13).

Hyperlipidemia and dyslipidemia are known risk factors for Bichat's tunic injuries, artery stenosis and atherosclerosis (19). Of note, $40-80 \%$ patients are reported to have hyperlipidemia 
A
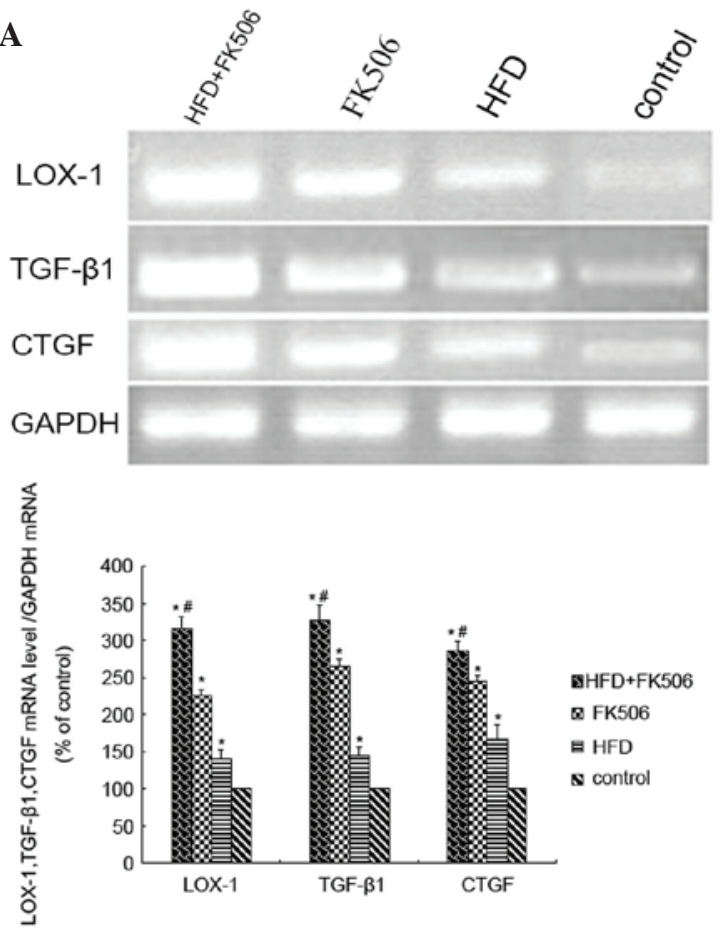

B

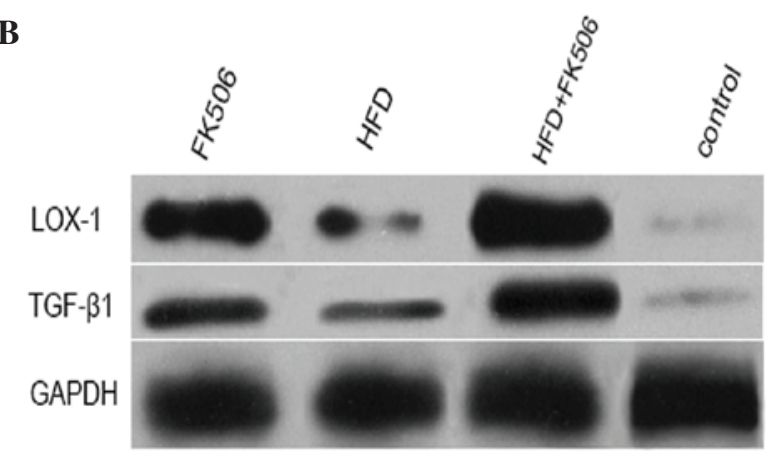

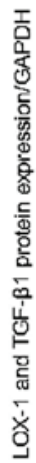

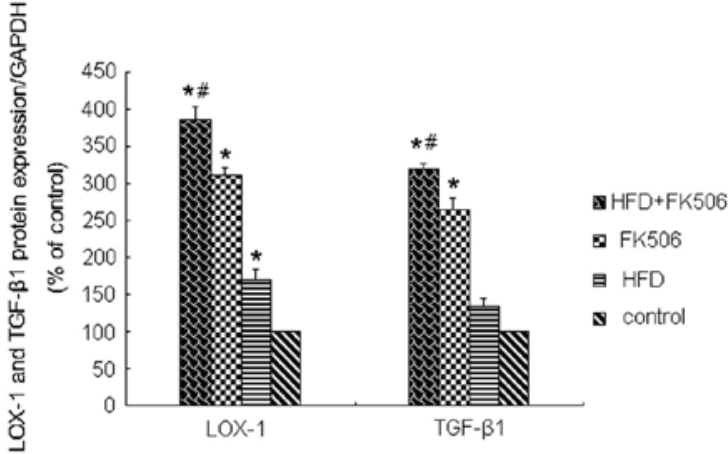

Figure 9. mRNA levels of LOX-1, TGF- $\beta 1$ and CTGF in the groups treated with HFD+FK506 (0.15 mg/kg/day) for 4 weeks, FK506 (0.15 mg/kg/day) for 4 weeks and HFD for 4 weeks. (B) Protein levels of LOX-1 and TGF- $\beta 1$ in the groups treated with HFD+FK506 (0.15 mg/kg/day) for 4 weeks, FK506 (0.15 mg/kg/day) for 4 weeks, and HFD for 4 weeks. "P<0.01 vs. control group; ${ }^{\#} \mathrm{P}<0.05$ vs. FK506 or HFD groups (3 independent experiments). HFD, high fat diet; FK506, tacrolimus; LOX-1, lectin-like oxidized low-density lipoprotein receptor- 1 ; TGF- $\beta 1$, transforming growth factor- $\beta 1$; CTGF connective tissue growth factor.

following renal transplantation (3,7). In a previous statistical study of 42 patients with CRAD, their blood lipid levels were found to be significantly higher, compared with those of other renal transplant patients without CRAD symptoms (20). In a follow-up clinical study of 28 patients with CRAD, diagnosed with kidney puncture biopsy, the Baff grades of the patients were positively correlated with renal function and blood lipid levels (21). Therefore, it is suggested that hyperlipidemia and dyslipidemia are important in the development of CRAD as non-immunological factors.

LDL and its bioactive oxidized form, ox-LDL, are essential in lipid metabolism. LOX-1, the major receptor of ox-LDL, can accelerate the development of CRAD following renal transplantation (8-10). LOX-1 was first identified as the receptor of ox-LDL in 1997 by Sawamura (22). Studies have revealed that, in certain pathological circumstances, including hyperlipidemia and atherosclerosis, LOX-1 is expressed at high levels not only in aortas, carotid arteries, thoracic aortas, coronary arteries and the venous plexus, but also in macrophages, smooth muscle cells, fibroblasts and platelets (23-25). LOX-1 is able to bind to and assist in the digestion of ox-LDL, which is recognized by other signaling molecules and induces the dysfunction or apoptosis of vascular endothelial cells (26). LOX-1 is expressed at high levels in atherosclerotic plaques and atherosclerotic plaque-derived cells, and its expression can be induced by specific proinflammatory or pro-atherosclerotic cytokines $(23,24)$. Therefore, LOX-1 is crucial to the development of several CVDs, including atherosclerosis and hypertension (10).

Post-transplantation dyslipidemia can be achieved by a number of factors, and the application of immunosuppressors, including FK506, are likely to be the most important (16). At present, the exact mechanism underlying FK506 toxicity remains to be fully elucidated, although lipid peroxidation and excess ROS production due to oxidative stress are known to be important in this process $(12,16)$. The results of the present study were consistent with this, which showed that high levels of ROS and hydrogen peroxide were produced following treatment, which was particularly true of ox-LDL following FK506 combination treatment. In a previous study, Apanay et al used different doses of Cs11A to treat transplant patients for 2 months, and found that the levels of ox-LDL increased significantly at high and normal doses of Cs11A, compared with low doses (27). Similar results were obtained in the present study, indicating that the effect of FK506 on the oxidative stress response was enhanced by high-fat exposure and vice versa. The high levels of ox-LDL resulting from this enhancement may cause irreversible damage to the blood vessel walls of transplanted grafts.

The importance of TGF- $\beta 1$ and its downstream gene, CTGF, is well established. TGF- $\beta 1$ is overexpressed in early fibrosis, and induces the expression of CTGF to maintain fibrosis, which is associated with CRAD $(28,29)$. Following exposure to a high-fat diet, increased levels of ox-LDL and LOX-1 can promote the expression of TGF- $\beta 1$ and CTGF (30). In the present study, $\mathrm{H} \& \mathrm{E}$ and Masson's trichrome staining showed kidney injury and the enhancement of fibrosis following exposure to a high-fat diet and FK506 treatment, which worsened over time. The expression levels determined in vivo and in vitro provided additional evidence that high-fat exposure and FK506 were able to increase the expression levels of TGF- $\beta 1$ and CTGF, with the most marked fibrosis 
developing following combined high-fat diet+FK506 treatment. The above results suggested that fibrosis genes can function to maximum extent under such circumstance and cause marked damage to renal grafts. Furthermore, the results of the present study demonstrated that siRNA against LOX-1 inhibited the synthesis of TGF- $\beta 1$, demonstrating that LOX-1 was capable of regulating TGF- $\beta 1$. Ox-LDL can promote levels of fibrinogen and increase the risk of allograft fibrosis through a synergistic mechanism (31). These findings may provide further information to assist in the therapy of post-renal transplant fibrosis.

As common complications of renal transplantation, hyperlipidemia and dyslipidemia severely impair long-term survival rates of patients. At present, increasing evidence supports the hypothesis that immunosuppressants, particularly glucocorticoids or cyclosporin, are critical in dyslipidemia, resulting in CVD and CRAD following transplantation $(17,32,33)$. However, the underlying mechanism remains to be fully elucidated. How ox-LDL and LOX-1 function in these processes also remains to be fully elucidated, as they are involved in multiple signaling pathways. Therefore, further investigations are required to determine the mechanism of immunosuppressant-induced hyperlipidemia or dyslipidemia resulting in CRAD. This may assist in the development of future therapies to reduce the occurrence of CVD or CRAD following renal transplantation, and prolong renal transplant graft and patient survival rates.

\section{Acknowledgements}

This study was supported by the National Natural Science Foundation of China (grant no. 2012sz0026), the Technology Support Plan of Sichuan Province (grant no. 2014SZ0210) and the Scientific Research Projects of Sichuan Health Bureau (grant no. YN 20140030).

\section{References}

1. Womer KL, Vella JP and Sayegh MH: Chronic allograft dysfunction: Mechanisms and new approaches to therapy. Semin Nephrol 20: 126-147, 2000.

2. Hernandez-Fuentes MP and Lechler RI: Chronic graft loss. Immunological and non-immunological factors. Contrib Nephrol 146: 54-64, 2005

3. Divakar D, Bailey RR, Frampton CM, George PM, Walmsley TA and Murphy J: Hyperlipidemia in stable renal transplant recipients. Nephron 59: 423-428, 1991.

4. Roodnat JI, Mulder PG, Zietse R, Rischen-Vos J, van Riemsdijk IC, IJzermans JN and Weimar W: Cholesterol as an independent predictor of outcome after renal transplantation. Transplantation 69: 1704-1710, 2000.

5. Serón D, Moreso F, Ramón JM, Hueso M, Condom E, Fulladosa X, Bover J, Gil-Vernet S, Castelao AM, Alsina J and Grinyó JM: Protocol renal allograft biopsies and the design of clinical trials aimed to prevent or treat chronic allograft nephropathy. Transplantation 69: 1849-1855, 2000.

6. Wissing KM, Abramowicz D, Broeders N and Vereerstraeten P: Hypercholesterolemia is associated with increased kidney graft loss caused by chronic rejection in male patients with previous acute rejection. Transplantation 70: 464-472, 2000.

7. Friemann S, Feuring E, Padberg W and Ernst W: Improvement of nephrotoxicity, hypertension, and lipid metabolism after conversion of kidney transplant recipients from cyclosporine to tacrolimus. Transplant Proc 30: 1240-1242, 1998.

8. Vela CG, Cristol JP, Descomps B and Mourad G: Prospective study of lipid disorders in FK506-versus cyclosporine-treated renal transplant patients. Transplant Proc 32: 398, 2000.
9. Ruan XZ, Varghese Z, Fernando R and Moorhead JF: Cytokine regulation of low-density lipoprotein receptor gene transcription in human mesangial cells. Nephrol Dial Transplant 13: 1391-1397, 1998.

10. Chen M, Masaki T and Sawamura T: LOX-1, the receptor for oxidized low-density lipoprotein identified from endothelial cells: Implications in endothelial dysfunction and atherosclerosis. Pharmacol Ther 95: 89-100, 2002.

11. Shihab FS, Bennett WM, Tanner AM and Andoh TF: Mechanism of fibrosis in experimental tacrolimus nephrotoxicity. Transplantation 64: 1829-1837, 1997.

12. Khanna AK and Pieper GM: NADPH oxidase subunits (NOX-1, p22phox, Rac-1) and tacrolimus-induced nephrotoxicity in a rat renal transplant model. Nephrol Dial Transplant 22: 376-385, 2007.

13. Kobashigawa JA and Kasiske BL: Hyperlipidemia in solid organ transplantation. Transplantation 63: 331-338, 1997.

14. Cheung CY, Wong KM, Chan HW, Liu YL, Chan YH, Wong HS, Chak WL, Cho KS, Chau KF and Li CS: Paired kidney analysis of tacrolimus and cyclosporine microemulsion-based therapy in Chinese cadaveric renal transplant recipients. Transpl Int 19: 657-666, 2006.

15. Ekberg H, Bernasconi C, Tedesco-Silva H, Vitko S, Hugo C, Demirbas A, Acevedo RR, Grinyó J, Frei U, Vanrenterghem Y, et al: Calcineurin inhibitor minimization in the Symphony study: Observational results 3 years after transplantation. Am J Transplant 9: 1876-1885, 2009.

16. Li HY, Li B, Wei YG, Yan LN, Wen TF, Zhao JC, Xu MQ, Wang WT, Ma YK and Yang JY: Higher tacrolimus blood concentration is related to hyperlipidemia in living donor liver transplantation recipients. Dig Dis Sci 57: 204-209, 2012.

17. Livak KJ and Schmittgen TD: Analysis of relative gene expression data using real-time quantitative PCR and the 2(-Delta Delta C(T)) Method. Methods 25: 402-408, 2001.

18. Racusen LC, Halloran PF and Solez K: Banff 2003 meeting report: New diagnostic insights and standards. Am J Transplant 4: 1562-1566, 2004.

19. Mango R, Clementi F, Borgiani P, Forleo GB, Federici M, Contino G, Giardina E, Garza L, Fahdi IE, Lauro R, et al: Association of single nucleotide polymorphisms in the oxidised LDL receptor 1 (OLR1) gene in patients with acute myocardial infarction. J Med Genet 40: 933-936, 2003.

20. Dimény E, Fellström B, Larsson E, Tufveson G and Lithell H: Hyperlipoproteinemia in renal transplant recipients: Is there a linkage with chronic vascular rejection? Transplant Proc 25: 2065-2066, 1993.

21. Dimény E, Wahlberg J, Lithell H and Fellström B: Hyperlipidaemia in renal transplantation-risk factor for long-term graft outcome. Eur J Clin Invest 25: 574-583, 1995.

22. Sawamura T, Kume N, Aoyama T, Moriwaki H, Hoshikawa H, Aiba Y, Tanaka T, Miwa S, Katsura Y, Kita T and Masaki T: An endothelial receptor for oxidized low-density lipoprotein. Nature 386: 73-77, 1997.

23. Chen M, Kakutani M, Minami M, Kataoka H, Kume N, Narumiya S, Kita T, Masaki T and Sawamura T: Increased expression of lectin-like oxidized low density lipoprotein receptor-1 in initial atherosclerotic lesions of Watanabe heritable hyperlipidemic rabbits. Arterioscler Thromb Vasc Biol 20: 1107-1115, 2000.

24. Kataoka H, Kume N, Miyamoto S, Minami M, Moriwaki H, Murase T, Sawamura T, Masaki T, Hashimoto N and Kita T: Expression of lectinlike oxidized low-density lipoprotein receptor-1 in human atherosclerotic lesions. Circulation 99: 3110-3117, 1999.

25. Draude G, Hrboticky N and Lorenz RL: The expression of the lectin-like oxidized low-density lipoprotein receptor (LOX-1) on human vascular smooth muscle cells and monocytes and its down-regulation by lovastatin. Biochem Pharmacol 57: 383-386, 1999 .

26. Cominacini L, Rigoni A, Pasini AF, Garbin U, Davoli A, Campagnola M, Pastorino AM, Lo Cascio V and Sawamura T: The binding of oxidized low density lipoprotein (ox-LDL) to ox-LDL receptor-1 reduces the intracellular concentration of nitric oxide in endothelial cells through an increased production of superoxide. J Biol Chem 276: 13750-13755, 2001.

27. Apanay DC, Neylan JF, Ragab MS and Sgoutas DS: Cyclosporine increases the oxidizability of low-density lipoproteins in renal transplant recipients. Transplantation 58: 663-669, 1994. 
28. Leask A: Transcriptional profiling of the scleroderma fibroblast reveals a potential role for connective tissue growth factor (CTGF) in pathological fibrosis. Keio J Med 53: 74-77, 2004.

29. Cheng O, Thuillier R, Sampson E, Schultz G, Ruiz P, Zhang X, Yuen PS and Mannon RB: Connective tissue growth factor is a biomarker and mediator of kidney allograft fibrosis. Am J Transplant 6: 2292-2306, 2006.

30. Sohn M, Tan Y, Wang B, Klein RL, Trojanowska M and Jaffa AA: Mechanisms of low-density lipoprotein-induced expression of connective tissue growth factor in human aortic endothelial cells. Am J Physiol Heart Circ Physiol 290: H1624-H1634, 2006.
31. Jin S, Mathis AS, Rosenblatt J, Minko T, Friedman GS, Gioia K, Serur DS and Knipp GT: Insights into cyclosporine A-induced atherosclerotic risk in transplant recipients: Macrophage scavenger receptor regulation. Transplantation 77: 497-504, 2004.

32. Massy ZA: Hyperlipidemia and cardiovascular disease after organ transplantation. Transplantation 72: S13-S15, 2001.

33. Varghese Z, Fernando RL, Turakhia G, Psimenou E, Fernando ON, Sweny P, Powis SH and Moorhead JF: Calcineurin inhibitors enhance low-density lipoprotein oxidation in transplant patients. Kidney Int Suppl 71: S137-S140, 1999. 